\title{
Gesundheit in der Gegenwart der Ahnen: Afrikanisches Heilen zwischen Akzeptanz und Ablehnung am Beispiel Südafrikas
}

\begin{abstract}
Health in the Presence of the Ancestors: African Healers between Acceptance and Denial: A Case Study from South Africa. Health and well-being for all is the ambitious aim of the third of the Sustainable Development Goals (SDGs) of the United Nations (UN). The no less ambitious definition of health of the World Health Organization (WHO) defines that health is a state of complete physical, mental and social well-being and not merely the absence of disease or infirmity. While in biomedical contexts treatment concentrates on physical healing, in the South African context the idea of healing and well-being subsumes a combination of physical, mental and social treatment and includes the ancestors and medicinal plants as an important category in the healing process. The ancestors in particular are representatives of the social past that reaches into the present. Healing as such has a multifaceted dimension even beyond the definition of health as proposed by the WHO.
\end{abstract}

\section{Einleitung}

„Gesundheit und Wohlergehen für alle“ heißt das dritte der 17 Nachhaltigkeitsziele (Sustainable Development Goals, SDGs) der Vereinten Nationen (UN). Ein hochgestecktes Ziel, gemessen an der Definition der Weltgesundheitsorganisation (WHO), die Gesundheit als „Zustand vollkommenen körperlichen, geistigen und sozialen Wohlbefindens und nicht allein das Fehlen von Krankheit und Gebrechen“ (WHO 2001) beschreibt, und gemessen an der oftmals schweren Umsetzbarkeit in Ländern, in denen die Gesundheitsversorgung bereits am Zugang zu grundlegender medizinischer Allgemeinversorgung scheitert. Das Streben nach Gesundheit und Gesunderhaltung ist jedoch, mit oder ohne Zugang zu medizinischer Versorgung, ein zentrales Anliegen in allen Regionen und Kulturen dieser Welt. So hat jede Kultur ihr eigenes, an die Umwelt und sozialen Bedingungen angepasstes, medizinisches System, das über Jahrtausende entwickelt, verfeinert und teilweise verschriftlicht wurde (Leslie 1976). Medizinische Systeme sind

Britta Rutert, Interdisziplinäre Arbeitsgruppe „Zukunft der Medizin: Gesundheit für alle“, Berlin-Brandenburgische Akademie der Wissenschaften

Ә OpenAccess. (C 2021 Britta Rutert, publiziert von De Gruyter. (cc) BY-NC-SA Dieses Werk ist lizenziert unter einer Creative Commons Namensnennung - Nicht kommerziell - Weitergabe unter gleichen Bedingungen 4.0 International Lizenz. https://doi.org/10.1515/9783110713336-013 
demnach gelebte Alltagssysteme, die z.B. durch äußere Einflüsse wie Migration kontinuierlichen Änderungen unterliegen.

In den Ländern des Globalen Nordens gilt die biomedizinische Versorgung als das primäre Versorgungssystem, jedoch erfreuen sich alternative Heilsysteme immer größerer Beliebtheit (Bodeker et al. 2005; Eisenberg et al. 1998). Yoga, Akupunktur oder Qi Gong sind weit verbreitete und genutzte therapeutische Praktiken, die von der WHO unter den Begriffen „Traditionelle, Komplementäre und Integrative Medizin“ zusammengefasst wurden (World Health Organization 2019). Deren salutogenetischer Zugang, also der prozessuale Zugang zu Gesundheit und Krankheit, grenzt sich stark ab vom pathologischen Zugang, der in der Medizin an den Universitäten gelehrt wird (Antonovsky \& Franke 1997). Traditionelle, Komplementäre und Integrative Medizin ermöglicht es dem Patienten, sich an der Heilung aktiv zu beteiligen und somit nicht alleine den physischen Heilungsprozess zu fördern, sondern auch die seelischen Prozesse zu integrieren. Trotz starker Diskussionen um die Wirksamkeit und Evidenz alternativer Heilmethoden wächst die Akzeptanz in Ländern des Globalen Nordens zunehmend. Je nach Krankheitsbild, wie zum Beispiel bei Krebserkrankungen, werden alternative Therapien komplementär zur biomedizinischen Versorgung gewählt (Coulter \& Willis 2007; Joos et al. 2008).

Auffällig ist, dass zwar asiatische Therapieansätze bekannt sind und häufig angewandt werden, dass in der Auswahl der therapeutischen Möglichkeiten aber selten Heilmethoden oder -mittel aus Afrika zu finden sind. Es gibt zwar einige Medizinalpflanzen, die nach strengen Qualitätsstandards den Markt der Europäischen Union oder der USA erreichen. Die Kapland-Pelargonie, die als Extrakt mit dem Namen Umckaloabo ${ }^{\circledR}$ in deutschen Apotheken verkauft wird, ist eine dieser Pflanzen (Morris 2012). Die Extrahierung und Synthetisierung einer chemischen Verbindung hat jedoch wenig mit dem Heilwesen in Afrika zu tun. Es sei denn, das Wissen von Heilerinnen oder Heilern wurde genutzt, um neue Heilpflanzen $\mathrm{zu}$ identifizieren und neue chemische Verbindungen $\mathrm{zu}$ identifizieren (Rutert 2020). Dieses als Bioprospektion beschriebene Vorgehen, die biologische und genetische Nutzbarmachung natürlicher Ressourcen (Reid et al. 1993), ist allerdings kein Spezifikum für Pflanzen aus Afrika, sondern wird von akademischen Institutionen und pharmazeutischen Unternehmen weltweit betrieben. Jenseits der im Handel erhältlichen Pflanzenprodukte ist jedoch wenig über afrikanische Heilsysteme bekannt. Sie haben eine globale Sonderstellung (Dilger et al. 2012) und scheinen sich wenig als Exportschlager für den Globalen Norden zu eignen. Woran liegt das? Ein Grund mag in dem von medialen Eindrücken unterstützten negativ beladenen Bild Afrikas als Kontinent voller Seuchen, Hunger, Armut, Krieg und ökonomischer Defizite liegen (Guest 2010). Dabei wird wenig differenziert, um welchen Teil Afrikas es sich handelt und inwieweit diese 
Stereotype dem realen Bild entsprechen. Zudem sind afrikanische medizinische Praktiken sowie der Gebrauch von Heilpflanzen aufgrund der meist regional orientierten, mündlichen Wissensübertragung und des Fehlens einer einheitlichen afrikanischen Sprache kaum dokumentiert (Ngubane 1977; Thornton 2017).

Was ist also das Spezifische an der afrikanischen Heilkunst und was bedeutet Gesundheit in diesem Kontext? Wieso sind afrikanische Heilpraktiken kaum im globalen medizinisch-therapeutischen Markt zu finden? Dieser Artikel beschreibt mit einem Blick auf die Region Kruger to Canyon (K2C) im Nord-Osten Südafrikas die Komplexität afrikanischer Heilsysteme als an regionale Bedingungen geknüpft, die trotz starker Widersprüchlichkeiten ein wesentlicher Teil der Kultur des schwarzen Südafrika sind.

\section{Traditionelles Heilen in Südafrika am Beispiel der Region Kruger to Canyon (K2C)}

Südafrika ist global-politisch gesehen eines der Länder mit der größten Schere zwischen Arm und Reich. Diese Situation bildet sich auch im öffentlichen Gesundheitswesen und der primären Gesundheitsversorgung ab. Obwohl mit dem Ende der Apartheid viel in Gesetze zur Umsetzung von zugänglicher und bezahlbarer Gesundheitsversorgung investiert wurde, bleibt die reale Situation polarisierend zwischen arm und reich, schwarz und weiß (Ataguba et al. 2014; Heaton \& Amoateng 2007). Auch wenn die von der UN propagierte Idee der universalen Gesundheitsversorgung in der Südafrikanischen Regierung Gehör findet und mit der National Health Insurance (NHI) ein Plan zur aktiven Umsetzung entwickelt wurde, bleibt diese doch weiter hinter den Erwartungen zurück. Was bedeutet das nun in Bezug auf die Gesundheit, insbesondere der ländlichen, schwarzen Bevölkerung?

Die ländlichen, abgeschnittenen Gebiete Südafrikas wurden während der Apartheid-Ära (1948-1994) in sogenannte Homelands unterteilt. Insgesamt gab es zehn Homelands, die jeweils einer ethnischen Gruppe zugewiesen wurden. Während die Homelands mit dem Ende der Apartheid 1994 aufgelöst wurden, sind deren fiktiven Grenzen teilweise bestehen geblieben. Bis heute sind die Regionen der ehemaligen Homelands dicht besiedelt und von Arbeitslosigkeit, Armut, schlechter Infrastruktur und Bildung geprägt. Ohne Arbeitsmigration in die großen Städte Durban, Kapstadt und Johannesburg wären diese Regionen bis heute abgeschnitten von ökonomischen, technologischen oder kulturellen Entwicklungen. Nach wie vor gibt es vielerorts kaum Zugang zu Elektrizität oder fließendem Wasser, Schulen sind schlecht ausgestattet und Gesundheitszentren oder 
Kliniken sind nur schwer zugänglich. Diese Lebenssituation steht in dem immer noch stark segregierten Land oft im Kontrast zu den Regionen, die von der weißen Bevölkerung bewohnt werden, wo gut ausgestattete Schulen und Krankenhäuser zur Lebensrealität gehören. Dieser Kontrast ist besonders in der Region Kruger to Canyon (K2C) in den Provinzen Mpumalanga und Limpopo sichtbar. Die K2CRegion, ein von der UNESCO erklärtes Bioreservat, befindet sich im sogenannten Lowveld, einem für lange Zeit von Malaria und Schlafkrankheit durchseuchten Gebiet. Weiße Siedler haben sich im höher gelegenen, malariafreien TransvaalGebiet niedergelassen. Die Grenze zwischen diesen beiden historisch markierten Gebieten ist bis heute sichtbar. Während die von weißen Farmern und Plantagenbesitzern bewohnte Region von weiten Feldern, Straßen ohne Schlaglöcher und einer definierten Ordnung bestimmt ist, ist die Region, in der die schwarze Bevölkerung lebt, von teils unbefahrbaren Straßen, ungeordneter Bebauung aus fertigen und halbfertigen Häusern und Hütten, Ziegen, Hunden und Kühen auf den Straßen und Kleinhändlern an den Straßenrändern bestimmt. Diese Region war auch als ehemaliges Homeland Gazankulu der Shangaan sprechenden Bevölkerung zugeordnet. Die Region ist heute eine der am dichtesten besiedelten, ärmsten und ethnisch diversesten Regionen Südafrikas (Thornton 2002). Die medizinische Versorgung beschränkt sich auf Mindeststandards in den Krankenhäusern der größeren Städte und auf Gesundheitszentren in den kleineren Ortschaften. Bei chronischen Krankheiten wie Diabetes sind diese Kliniken wegen mangelhafter Ausstattung, hoher Kosten der medizinischen Versorgung und schweren Erreichbarkeit für die lokale Bevölkerung kaum hilfreich.

In einer solchen Situation sind lokale traditionelle Heiler und Heilerinnen unentbehrlich. Sie sind oft der einzige Zugang zu physischer wie psychischer Gesundheitsversorgung. Viele Menschen suchen je nach Erkrankung sowohl Kliniken und Gesundheitszentren als auch Heiler auf (Pinkoane et al. 2012). Eine Integration von biomedizinischer Versorgung und traditionellem Heilen ist von der Südafrikanischen Regierung mit dem Ende der Apartheid politisch gefördert worden (Mokgobi 2013; Pinkoane et al. 2012). Da in abgelegenen Regionen Kliniken oft unerreichbar sind, sind Heiler die erste und einzige Wahl. Sie werden bei diversen Krankheitsformen konsultiert, von Bauchschmerzen bis zu chronischen Krankheiten, aber auch bei z. B. sozialen oder familiären Konflikten. Die Anzahl der Heiler in einer so dicht besiedelten Region wie der K2C-Region ist schwer zu bestimmen, da die Heiler-Ausbildung weder professionell strukturiert ist noch staatlich kontrolliert wird. Zwar versucht das Südafrikanische Gesundheitsministerium eine Registrierungspflicht für Heiler einzuführen, die Umsetzung gestaltet sich jedoch aufgrund der hohen Dichte illegal in der Region lebender Menschen und der hohen Analphabeten-Rate schwierig. Eine Professionalisierung wird dennoch von vielen Heiler-Organisationen begrüßt, auch um die Pro- 
fession des Heilers so zu sichern. Traditionelles Heilen ist zwar eine zutiefst spirituelle Tätigkeit, aber auch ein Geschäft. In einer von hoher Arbeitslosigkeit betroffenen Gegend wie der K2C-Region kann der Beruf des Heilers das Überleben ganzer Familien sichern. Ursprünglich ist das Heilwesen jedoch keine Tätigkeit, die man wie Akupunktur erlernen kann. Zum Heiler wird man durch die Ahnen berufen.

\section{3 „The calling“: Berufung als Heilerin oder Heiler}

„Ich wurde plötzlich blind“, „Ich hatte plötzlich meine Periode nicht für mehrere Monate“ oder „über einen langen Zeitraum gab es viele Unglücke und Todesfälle in meiner Familie“, das waren Beweggründe, die Menschen dazu veranlasst haben, einen Heiler aufzusuchen. Während der Konsultationen stellte sich heraus, dass der Grund für Krankheiten und soziales Unglück darin zu suchen sei, dass die Ahnen wollten, dass der Patient ein Training zum Heiler absolviere (ukuthwasa). ${ }^{1}$ Der Ruf zum Heiler spielt besonders dann eine Rolle, wenn es sich um spirituelles Heilen handelt. Heiler, die mit Hilfe von Divination und einer spirituellen Verbindung zu den Ahnen arbeiten, werden Sangoma genannt. Während der Konsultation bei einem Sangoma wird klar, dass bei der physischen oder sozial erkrankten Person ein Ruf zum Heiler vorliegt. Allerdings endet nicht jede Konsultation bei einem Sangoma damit, dass der Patient selber Heiler werden muss. Der Ruf ist vielmehr ein Aushandlungsprozess zwischen dem Patienten und den Ahnen, bei dem der Heiler helfend zur Seite steht. Oftmals wehren sich die Auserwählten, den Ruf als Heiler anzunehmen. Das Training erfordert viele Entbehrungen. Nicht nur müssen sich die Initianten für Monate oder sogar Jahre von ihren Familien trennen, um in der Familie des trainierenden Heilers zu leben. Auch sind mit dem Training strikte Nahrungs- und Handlungsvorschriften verbunden. Eier, Milch, Zucker oder Alkohol sind verboten, genauso sexuelle Handlungen jeglicher Art. Es dürfen nur bestimmte Kleidungsstücke getragen werden, je nach Tradition in Weiß oder Rot. Ferner kostet die Ausbildung viel Geld. Viele Initianden müssen sich daher Geld von Familienmitgliedern oder Freunden leihen, in armen Regionen ist das nicht einfach. Das liegt nicht nur an mangelnden finanziellen Ressourcen, sondern auch daran, dass traditionelles Heilen selbst in der eigenen Kultur auf ein ambivalentes Verhältnis von Zustim-

1 Ukuthwasa heisst „to become visible“ (Hammond-Tooke 1998) Es wird auch als „The sickness of the calling“ (die Krankheit zur Berufung) bezeichnet (Berlund 1976; Buhrmann 1994; Wreford 2004). Es ist ein Ruf, der die Person dazu führt, in die Rolle des Heilers initiiert zu werden (Hirst 1990). 
mung und Ablehnung stößt. Auf der einen Seite ist das traditionelle Heilen tief verankert im kulturellen Leben der Menschen, auf der anderen Seite sind die oft sehr christlich geprägten ländlichen Regionen Südafrikas bestrebt, Aberglauben und Hexerei zu unterbinden. Beide Fähigkeiten werden Heilern zugesprochen. Auch von Seiten biomedizinischer Ärzte gibt es Kritik an traditionellen Heilern, insbesondere, wenn diese sich als Wunderheiler von Krankheiten wie HIV/AIDS ausgeben. Im nächsten Abschnitt wird diese Ambivalenz näher beleuchtet.

\section{Ambivalente Gefühle: Traditionelles Heilen in Afrika}

„We are healers, not witches“, sagte mein Forschungsassistent und ausgebildeter Heiler, Rodney Sibuyi, immer wieder während meines Forschungsaufenthaltes (2011-12) in der K2C-Region. In Südafrika werden traditionelles Heilen und Muthi, die von Sangoma benutzen Substanzen wie Medizinalpflanzen, Rinden, Tierkörperteile, Steine oder Metalle, sehr widersprüchlich gesehen. Sangoma können sowohl als Heiler arbeiten als auch Hexerei ausüben. In den Tageszeitungen sind häufig Schlagzeilen über „muthi-murder“ oder „black magic with muthi“ zu lesen. Die positive wie die negative Ausübung des Heilens gehören unmittelbar zusammen in der öffentlichen Wahrnehmung. Die Angst vor Hexerei ist groß, zugleich gibt es aber einen unumstößlichen Glauben an die Kraft der Heiler und deren Muthi. Heiler werden zu Rate gezogen, auf kleinster lokaler Ebene genauso wie von Mitgliedern der Regierung und Ihre guten wie weniger guten Ratschläge werden befolgt. Ein verheerendes Beispiel eines weniger guten Rats eines Heilers war, den Aufstand der Mienenarbeiter der Marikana-Miene bei Johannesburg, bekannt als das Marikana massacre, mithilfe eines Muthi zu begehen. Mit einer von dem Heiler produzieren Muthi-Mischung eingerieben wären die Aufständischen gefeit gegen Angriffe durch Gewehrkugeln und daher unbesiegbar. Der Aufstand endete katastrophal, es wurden 34 Mienenarbeiter durch die Maschinengewehrkugeln der Polizei getötet. Die Muthi-Mischung zeigte keinerlei Wirkung (Conway-Smith 2012).

Diese nach missbräuchlicher Ausübung klingende Seite des traditionellen Heilens wird aber konterkariert durch eine tiefe historische Manifestation des Heilwesens in lokalen Strukturen (Flint 2004). Dieses Heilwesen ist geprägt von soziokulturellem und spirituellem Wissen und einem tiefen Verständnis für die lokale Umwelt und Biodiversität. Insbesondere das Wissen über Medizinalpflanzen ist abhängig vom lokalen Pflanzenbestand. Heiler bezeichnen sich entsprechend als „members of [a] profession with distinct intellectual tradition“ 
(Thornton 2009). In den dicht besiedelten Dörfern der K2C-Region leben große Familien auf engstem Raum und in direktem Kontakt zum nächsten Nachbarn. Neben den Kirchen gibt es kaum Anlaufstelle für soziale und/oder psychische Probleme. Heiler werden daher besonders bei sozialen und familiären Krisen als neutrale und klärende Instanz aufgesucht. Ähnlich wie medizinisch ausgebildete Ärzte haben auch Heiler einen Code of Conduct, der unter anderem Verschwiegenheit impliziert (Rutert 2020).

Heiler-Traditionen in Südafrika unterscheiden sich regional. Was sie aber alle verbindet, ist die tiefe Verbindung zu und mit den Ahnen. Die Ahnen spielen grundsätzlich eine große Rolle im Leben des schwarzen Südafrika, die Verbindung eines Heilers mit den Ahnen ist von tief spiritueller Bedeutung. Diese Verbindung wird während des Trainings zum Heiler manifestiert und bleibt während des gesamten Heilerlebens bestehen. Sie ist die Grundlage des Heilens und bildet die zentrale Verbindung zwischen Heiler und Patient. Jeder Heiler bekommt eine bestimmte Begabung und Fähigkeiten durch die Ahnen mitgegeben. Mein Forschungsassistent hatte besondere Fähigkeiten in der Technik des Knochen-Werfens und Interpretierens (tinhlolo). Dabei setzt sich der Heiler in seinem heiligen Raum (Indumba) dem Patienten gegenüber und tritt somit mit dem Patienten und den Ahnen in Verbindung. Der Heiler nimmt einen Beutel, gefüllt mit diversen Knochen, Muscheln, Geldstücken und anderen Gegenständen. Er schüttelt den Beutel und wirft die Gegenstände vor sich auf ein Ziegenfell oder eine Strohmatte. Die Gegenstände nehmen auf der Matte eine zueinander in Beziehung stehende Position ein, so liegen z. B. zwei ähnlich aussehende Knochen nebeneinander oder eine Muschel liegt mit der geöffneten Seite nach oben. Jede Position der Gegenstände zueinander hat eine Bedeutung, die die soziale Realität des Patienten widerspiegelt. Während der Heiler diese Position deutet, spricht er mit dem Patienten in einer ritualisierten Form (jede Aussage endet mit dem Wort Siyavuma - „Wir stimmen zu“). Dabei beobachtet er den Patienten und dringt so immer tiefer ein in die sozialen Beziehungen des Patienten ein. Diese Interaktion wird oft von einer starken Emotionalität der Patienten begleitet.

Eine Patientin, die mit ihrer Tochter zum Kurieren ihrer chronischen Rückenschmerzen aus einer zwei Stunden entfernten Stadt zu ihm kam, ist während eines „Knochen-Werfen“-Rituals in dramatisches Schluchzen verfallen. Bald stellte sich heraus, dass der Mann der Patientin schon seit mehreren Jahren eine zweite Frau hatte. Obwohl es nicht unüblich ist im subsaharischen Afrika, dass Männer mehrere Frauen haben, wurde klar, dass dieser Mann seine Affäre gerne zur ersten Frau machen würde und dass damit die erste Frau ihre Rechte als Ehefrau verlieren würde, d.h. auch ihre Rechte an Haus und Hof. Der Streit zwischen den Eheleuten schwelte schon lange, und die Frau war sichtlich verzweifelt. Zur Heilung wurden vom Heiler dann verschiedene Schritte vorgeschlagen. Zu- 


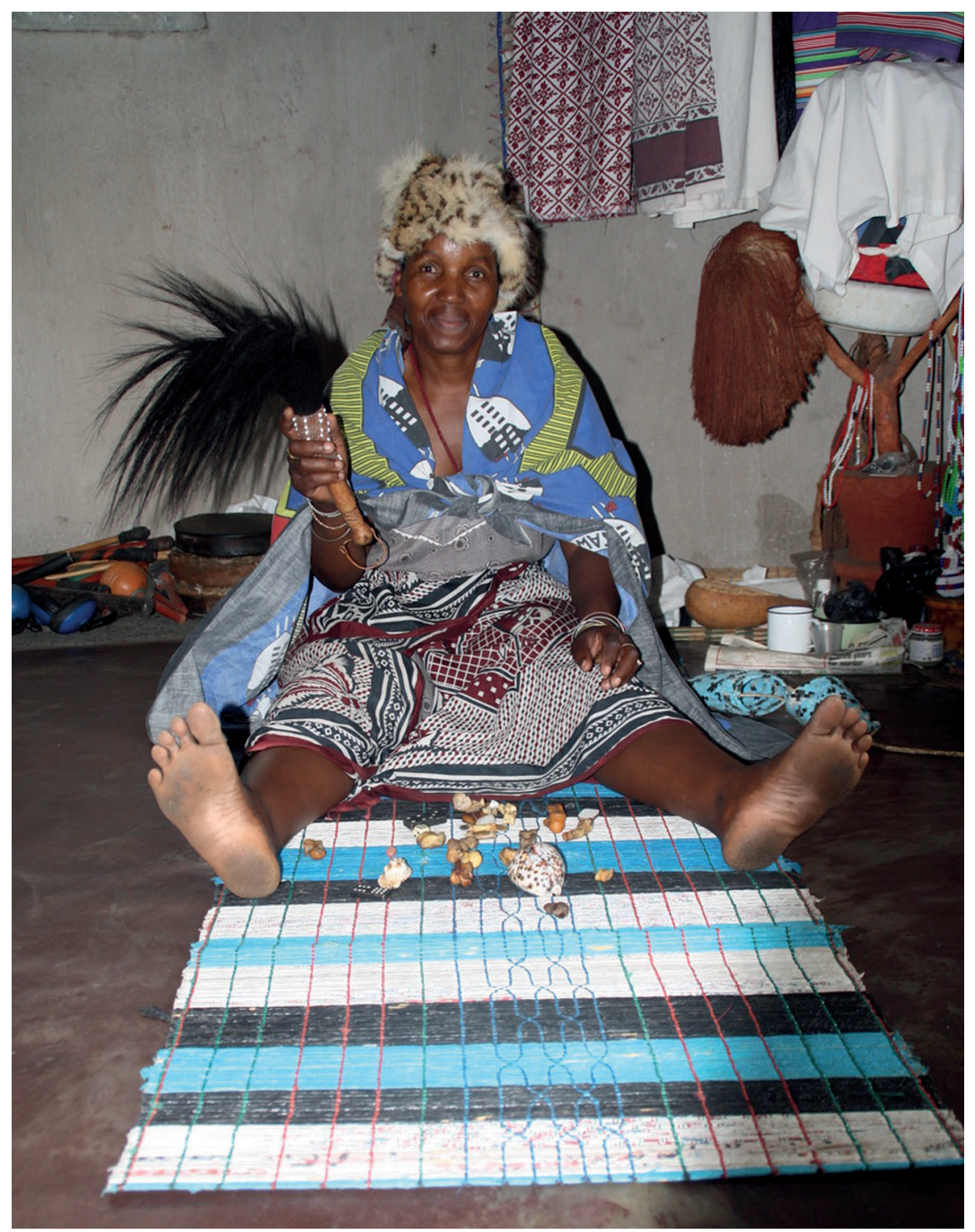

Abb. 1: Heilerin bei einem tinhlolo-Ritual. Foto: Britta Rutert

nächst muss die Patientin ein Dampfbad (Futha) mit ausgewählten Kräutern machen. Während dieser Futha soll sie sich ihre Wünsche und Ziele aufsagen. Nach der Futha erhält sie kleine Ritze, die mit einer Rasierklinge entlang der Schulter eingeritzt werden, genau an der Stelle, an der die Rückenschmerzen 
präsent sind. In die kleinen Ritze wird ebenfalls eine Muthi-Mischung eingerieben. Die Frau bekommt nach diesem Ritual noch eine Muthi-Mischung mitgegeben, die sie regelmäßig applizieren soll. Sie werde sehen, so der Heiler, ihr Mann werde sich am Ende für sie entscheiden. Sie soll dem Heiler später berichten, ob seine Behandlung Erfolg gezeigt hat. Die Behandlung kostete insgesamt 700 Südafrikanische Rand (damals ca. 50 Euro), eine Menge Geld für die Frau. Sie kann die Summe nicht auf einmal zahlen und verspricht, sie bald zu bringen.

\section{Was bedeutet Gesundheit im Kontext traditionellen Heilens in Südafrika?}

Auch wenn die Geschichte anekdotenhaft wirkt, so beschreibt sie doch die therapeutische Realität vieler Konsultationen zwischen Patient und Heiler. Der Heiler hat einen zweifachen Heilungsweg gewählt. Er hat die Rückenschmerzen der Patientin behandelt und gleichzeitig die psychosozialen Probleme mit Hilfe eines Rituals zunächst an die Oberfläche geholt und dann behandelt. Wichtig waren dafür das Gespräch, in dem die Patientin ihre Sorgen offenlegen konnte, sowie die rituelle Handlung am Körper. Während der gesamten Behandlungszeit ist die volle Aufmerksamkeit und Konzentration des Heilers auf die Patientin gerichtet gewesen. Für die Heilung wurde eine komplexe Verbindung zwischen dem Heiler und der Patientin, den Ahnen des Heilers und dem sozialen Umfeld der Patientin sowie der applizierten Muthi hergestellt. Der Glaube daran, dass dieses Interaktionsfeld eine heilende Bedeutung hat, ist hier essentiell. Diese Verbindung ist der Schlüssel zu Heilung und Wohlergehen. Robert Thornton beschreibt diese Verbindung folgendermaßen:

Bungoma, the knowledge and practice of ,traditional healing' in eastern Mpumalanga, is built on the fundamental premise that all persons are exposed to each other and to other person-like agents, including ancestors and witches, among others. This mutual and inescapable exposure is the condition for the possibility of healing but also ultimately the cause of all illness, misfortune and death. Against this, the sangoma as healer attempts to augment the self of the exposed being through protective magic and by exposing relations between tangible (living human) and intangible (spiritual) agents or persons. Bungoma comprises multiple modalities including trance, music and rhythm, divination, herbal lore, teaching and learning, craftsmanship and healing. The aim of bungoma is to enable patients to heal themselves by transforming their personal narratives of self (Thornton 2017, S. 1-43).

Was also bedeutet Gesundheit in diesem Kontext? Mit der Definition von Gesundheit der WHO ist der Terminus Gesundheit von der rein biomedizinischen, pathologisch orientierten Sicht entbunden worden und hat die seelische und 
geistige Dimension integriert. Wie diese seelisch und geistige Dimension aber genau aussieht, hat die WHO-Definition offengelassen. Die Einbeziehung der Ahnen als spirituelle Dimension könnte als die Dimension geistigen Wohlergehens interpretiert werden, und doch ist es eine Dimension, die global-politisch gesehen nicht gedacht wird, wenn es um Gesundheit geht. In den südafrikanischen Heiler-Traditionen sind die Ahnen jedoch die wesentliche Dimension, die Heilung herbeiführen. Auch wenn eine Konsultation nicht zu einem persönlichen Ruf zum Heiler führt, sondern zu einem Heilprozess wie bei der oben genannten Patientin, sind die Ahnen wesentlich. Oft müssen Opfertiere wie Hühner oder Ziegen von dem Patienten und dessen Familie geschlachtet werden, die den Ahnen geopfert werden, um Heilung auf physischer und psychischer Ebene zu erwirken. Sind die Ahnen nicht miteinbezogen und werden sie nicht beachtet, können sie den Menschen sogar Schaden zufügen. Gesundheit in diesem Kontext bedeutet also nicht nur das Fehlen von Krankheit und Gebrechen, sondern auch noch die Herstellung einer „gesunden Gemeinschaft“. Diese Gemeinschaft beinhaltet nicht nur die aktuelle Lebensgemeinschaft der Familie, des Klans oder der dörflichen Gemeinschaft, sondern auch die in diesen sozialen Kosmos integrierten Ahnen. Die südafrikanische Lebensphilosophie Ubuntu ${ }^{2}$ bezieht die Lebendigen wie die Toten mit ein, die materiellen und die nicht materiellen Kräfte. Somit wird eine holistische Sicht des Heilens hergestellt, die die Dimension der Vergangenheit durch die Ahnen, miteinbezieht. Durch die Verabreichung von Muthi wird zusätzlich die lokale Biodiversität integriert. Die Abbildung der Knochen beim „Knochen-Werfen“-Ritual zeigt plastisch diesen Kosmos sozialer Strukturen aus Vergangenheit, Gegenwart und Zukunft. Gesundheit ist daher nicht rein pathologisch, sondern bezieht auch die Dimensionen Zeit und Umwelt mit ein.

\section{Schlussbemerkungen}

Gesundheit ist ein komplexes Gebilde, das in der WHO Definition zu erfassen versucht wird. In der biomedizinischen Praxis wird vor allem Wert auf die körperliche Heilung gelegt. Bei Patienten entsteht dadurch aber häufig das Gefühl von mangelnder Zuwendung, der prozessualen Zugang zu Heilung, der die Selbstheilungskräfte miteinbezieht, wird als fehlend erachtet. Südafrikanisches

2 Ubuntu, in Xhosa mit „Umntu ngumntu ngabantu“ umschrieben, ist eine afrikanische Lebensphilosophie, die mit „eine Person ist eine Person durch andere Personen“ übersetzt werden kann (Tutu 1999). 
Heilen widmet sich der geistigen, emotionalen und vor allem sozialen Dimension des Heilens. Da körperliche Probleme und persönliche Konflikte stets in einer Einheit mit dem sozialen Gesamtgefüge gesehen werden, muss sowohl die Ebene der Lebenden als auch die der Ahnen miteinbezogen werden. Gesundheit ist daher nicht rein physisch zu betrachten, sondern beinhaltet stets das gesamte soziale Leben einer Person. Erst wenn dieses soziale Leben von Problemen befreit ist, kann Gesundung und Wohlergehen stattfinden. In diesem Sinne sind traditionelle Heiler nicht alleine für die physische Heilung wichtig, sondern auch für die Aufrechterhaltung der sozialen Ordnung. Da diese soziale Ordnung von guten wie weniger guten Kräften bestimmt ist, liegen Heilung und Hexerei nah beieinander und werden in den engen sozialen Verhältnissen in Südafrika situativ ausgetauscht. Dieses von Ambivalenzen und regionalen Bedingungen samt lokaler Umwelt und Biodiversität geprägte System kann kaum in andere Kontexte übertragen werden. Eine Ausbildung zum Heiler in einem Zentrum für afrikanisches Heilen in Berlin-Kreuzberg ist schon deshalb schwer vorstellbar, weil der soziale Kontext und die notwendigen natürlichen Ressourcen fehlen. Nicht zuletzt sind die archaisch anmutenden und streng hierarchischen Methoden während des Heiler-Trainings kaum über den südafrikanischen Kontext hinaus vermittelbar. Im Gegensatz zu der eher technischen Ausbildung z. B. in Akupunktur, die auf einem jahrhundertealten, verschriftlichen Wissenskorpus basiert gibt es für das südafrikanische Heilwesen keine „Gebrauchsanleitung“. Ausbildung und Heilung beruhen vielmehr auf intuitiv erfahrbarem und übertragbarem Wissen, denn auf schulischem, global transportierbarem Wissen.

\section{Literatur}

Antonovsky, Aaron/Franke, Alexa (1997): Salutogenese. Zur Entmystifizierung der Gesundheit. Tübingen: dgvt.

Ataguba, John E./Day, Candy/McIntyre, Di (2014): „Monitoring and Evaluating Progress towards Universal Health Coverage in South Africa“. In: PLOS Medicine 11(9), S. e1001686.

Berlund, Axel-Ivar (1976): Zulu Thoughts, Patterns and Symbolism. London: Hurst \& Co. Bodeker, Gerard/Ong, Chi-Keong/Grundy, Chris et al. (2005): WHO Global Atlas of Traditional, Complementary and Alternative Medicine. Kobe, Japan: WHO Centre for Health Development, https://apps.who.int/iris/handle/10665/43108, besucht am 6.1.2020.

Buhrmann, Vera (1994): Living in Two Worlds. Communication between a White Healer and Her Counterpart. Cape Town \& Pretoria: Human \& Rousseau.

Conway-Smith, Erin (2012): „South Africa: Striking Miners ,thought they were invincible“ after Taking ,muti““. https://www.pri.org/stories/2012-08-21/south-africa-striking-minersthought-they-were-invincible-after-taking-muti, besucht am 6.1.2020. 
Coulter, Ian/Willis, Evan (2007): „Explaining the Growth of Complementary and Alternative Medicine“. In: Health Sociology Review 16(3-4), S. 214-225.

Dilger, Hansjörg/Kane, Abdulaye/Langwick, Stacey (2012): Medicine, Mobility, and Power in Global Africa: Transnational Health and Healing Bloomington: Indiana University Press.

Eisenberg, David M./Davis, Roger B./Ettner, Susan L. et al. (1998): „Trends in Alternative Medicine Use in the United States, 1990-1997: Results of a Follow-up National Survey“. In: Jama 280(18), S. 1569-1575.

Flint, Karen (2004): Healing Traditions. African Medicine, Cultural Exchange, and Competetion in South Africa, 1820-1948. Athens: Ohio Press.

Guest, Robert (2010): The Shackled Continent: Power, Corruption, and African Lives. Washington: Smithsonian Books.

Hammond-Tooke, David W. (1998): „Selective Borrowing? The Possibility of San Shamanistic Influence on Southern Bantu Divination and Healing Practices“. In: The South African Archaeological Bulletin 53(167), S. 9-15.

Heaton, Tim B./Amoateng, Acheampong. Y. (2007): „The Family Context for Racial Differences in Child Mortality in South Africa“. In: A. Y. Amoateng/T. B. Heaton (Hrsg.): Families and Households in Post-apartheid South Africa: Socio-demographic Perspectives. Cape Town: HSRC Press, S. 171-187.

Hirst, Martin (1990): The Healer's Art: Cape Nguni Diviners in the Township of Grahamstown. Grahamstown: Grahamstown University.

Joos, Stefanie/Musselmann, Berthold/Miksch, Antje et al. (2008): „The Role of Complementary and Alternative Medicine (CAM) in Germany - A Focus Group Study of GPs“. In: BMC Health Services Research 8, S. 127-127.

Leslie, Charles (1976): Asian Medical Systems: A Comparative Study. Berkeley, CA: California University Press.

Mokgobi, Maboe G. (2013): „Towards Integration of Traditional Healing and Western Healing: Is This a Remote Possibility?“. In: African Journal for Physical Health Education, Recreation, and Dance 2013 (Suppl. 1), S. 47-57.

Morris, Christopher (2012): „Pharmaceutical Bioprospecting and the Law: The Case of Umckaloabo in a former Apartheid Homeland of South Africa“. In: Anthropology News 53(10), S. 6-7.

Ngubane, Heriette (1977): Body and Mind in Zulu Medicine: An Ethnography of Health and Disease in Nyuswa-Zulu Thought and Practice. London: Academic Press.

Pinkoane, Martha G./Greeff, Minrie/Koen, Magdalena P. (2012): „A Model for the Incorporation of the Traditional Healers into the National Health Care Delivery System of South Africa“. In: African Journal of Traditional, Complementary, and Alternative Medicines 9 (Suppl. 3), S. $12-18$.

Reid, Walter V. /Laird, Sarah A. /Meyer, Carrie A. et al. (1993): Biodiversity Prospecting. Washington: The World Reseach Institute.

Rutert, Britta (2020): Contested Properties: People, Plants, and Politics in Post-Apartheid South Africa. Bielefeld: Transcript.

Thornton, Robert (2002): „Environment and Land in Bushbuckridge, South Africa“. In: L. Zarsky (Hrsg.): Human Right and the Environment: Conflicts and Norms in a Globalizing World. Oxon \& New York: Earthscan.

Thornton, Robert (2009): „The Transmission of Knowledge in South African Traditional Healing“. In: Africa 79(1), S. 17-34. 
Thornton, Robert (2017): Healing the Exposed Being. Johannesburg: Wits University Press.

Tutu, Desmond (1999): No Future without Forgiveness. London: Image.

World Health Organization (2019): „The WHO Global Report on Traditional and Complementary Medicine 2019 is Released“. World Health Organization. https://www.who.int/news-room/ detail/20-05-2019-the-who-global-report-on-traditional-and-complementary-medicine2019-is-released, besucht am 6.1.2020.

Wreford, Jo Tboeka (2004): Working with Spirit. Experiencing Izangoma Healing in Contemporary South Africa. New York \& Oxford: Berghahn Books. 